\title{
Estudo de ondas estacionárias em uma corda com a utilização de um aplicativo gratuito para smartphones
}

(Study of stationary waves on a string with the use of a free application forsmartphones)

\author{
Anderson Guimarães Guedes ${ }^{\mathbb{m}}$ \\ Escola de Ciências e Tecnologia, Universidade Federal do Rio Grande do Norte, Natal, RN, Brasil \\ Recebido em 24/8/2014; Aceito em 25/3/2015; Publicado em 30/6/2015
}

\begin{abstract}
Este trabalho apresenta uma experiência de baixo custo de ondas estacionárias em uma corda. Ela usa um aplicativo de smartphone para sistema operacional Android, o "PA Tone", que gera um sinal senoidal ao invés de um caro gerador de ondas comercial. Utilizamos um osciloscópio para analisar a onda senoidal gerada a partir do aplicativo para verificar se houve alguma mudança na forma de onda com o aumento da freqüência. Também usamos um osciloscópio para verificar a confiabilidade dos valores de frequência indicados nas configurações do aplicativo. O gerador de sinais "PA Tone" foi então usado com um oscilador mecânico ligado a uma corda, no clássico experimento de criação de ondas estacionárias, no qual a densidade linear da corda pode ser inferida. Este experimento mostrou que o aplicativo "PA Tone" é uma alternativa viável ao gerador de sinais comercial. Palavras-chave: ondas estacionárias em uma corda, experimento de física de baixo custo, física com smartphones, simulador de sinais.
\end{abstract}

This work presents a low cost experiment of standing waves on a string. It uses the smartphone app for Android operating system, the "PA Tone", that generates a sinusoidal signal instead of the expensive commercial hardware. An oscilloscope was used to analyze the sine wave generated from the application to check if there was any change in the waveform with increasing frequency. We also used an oscilloscope to check the reliability of the frequency values indicated at the application settings. The signals generator "PA Tone" was then used with a mechanical oscillator attached to a string, in the classical experiment of creating standing waves, from which the linear density of the string can be inferred. This experiment showed that the smartphone app "PA Tone" is a viable alternative to the commercial signal generators.

Keywords: standing waves on a string, low cost physics experiment, physics with computers, signal simulator.

\section{Introdução}

A realidade de muitos laboratórios de física de nossas escolas de Ensino Médio é angustiante, os problemas vão desde espaços inadequados para fazer as atividades experimentais até a falta de equipamentos para a realização dos experimentos [1]. Uma das alternativas para contornar os problemas enfrentados pela falta de equipamentos nos laboratórios de física é a elaboração de experimentos de baixo custo.

A utilização de telefones modernos do tipo smartphones é bem comum e já faz parte do cotidiano de muitos professores e alunos. Os smartphones atuais podem ser considerados verdadeiros computadores de bolso, uma vez que, alguns têm poder de processamento e memória similares a computadores do tipo desktop. Outra caracterítica dos smartphones é a grande quantidade de aplicativos voltados para as mais variadas ne-

\footnotetext{
${ }^{1}$ E-mail: andersongguedes@yahoo.com.br.

cessidades. Sabendo das inúmeras aplicações dos celulares do tipo smartphone hoje em dia, resolvemos pesquisar aplicativos voltados para a geração de sinais em celulares e verificar a viabilidade de utilizá-los como um gerador de sinais em um experimento de baixo custo para estudo das ondas estacionárias em uma corda.

Segundo Cavalcante e Tavolaro [2] o objetivo de propor experimentos de baixo custo para estudo da onda estacionária seria contribuir para que professores do Ensino Médio tenham recursos educacionais que facilitem o entendimento de conceitos da física e, mais especificamente, os aspectos conceituais dos conteúdos de ondulatória e acústica.

O estudo da onda estacionária assistido por computador já foi discutido por alguns autores [2-4]. Em 2004 Cavalcante e Tavolaro [2] propõem uma configuração de experimento de baixo custo para estudo de ondas esta- 
cionárias constituído basicamente por um alto falante adaptado para fixação de um fio, uma caixa de som com controle de volume usada em PC's, pesos, polia, suportes, fio e o software "Sine Wave Generator". Mello [3] em 2007 sugeriu o uso do sofware Audio Sweep Gen (v. 2.2) como fonte de sinal senoidal e oscilador mecânico vibrando na vertical (Fig. 1). No ano de 2012, Cavalcante, Peçanha e Teixeira [4], em um artigo publicado na Revista Brasileira de Ensino de Física, sugeriram algumas modificações no experimento apresentado em 2004. As principais modificações foram na substituição do software gerador de sinais, onde também usaram o software Audio SweepGen, e na forma como foi disposto o oscilador mecânico (alto falante) no experimento, vibrando na direção vertical.

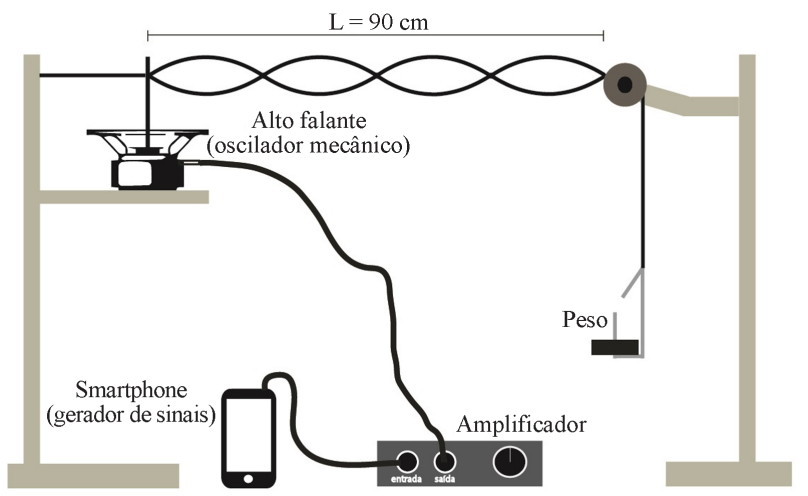

Figura 1 - Detalhes do experimento usado para estudar as ondas estacionárias em uma corda.

O objetivo deste trabalho é apresentar uma configuração de experimento para estudo das ondas estacionárias em uma corda, de baixo custo, e que tem como gerador de sinais um aplicativo para celular smartphone denominado "PA Tone". O trabalho também apresenta resultados comparativos de dados entre o aplicativo "PA Tone", um gerador de sinais comercial e o software Audio SweepGen.

\section{Ondas estacionárias em uma corda}

Quando vibrações são produzidas em uma corda esticada entre 2 pontos fixos produzimos ondas esta-

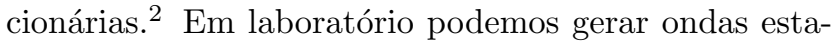
cionárias a partir de um oscilador mecânico (membrana de um alto falante), em que prendemos uma das extremidades de uma corda de comprimento $L$, tensionando com um peso a outra extremidade da corda (Fig. 1). Logo, se tivermos uma onda estacionária em uma corda, pode-se constatar facilmente que o comprimento $L$ será

$$
L=n \frac{\lambda}{2}
$$

em que lambda é o comprimento de onda e $n=1$, $2,3, \ldots$ Este resultado indica que apenas alguns modos de vibração na onda estacionária são permitidos numa corda com as extremidades fixas [5]. Os padrões de onda estacionária em uma corda são mostrados na Fig. 2 .

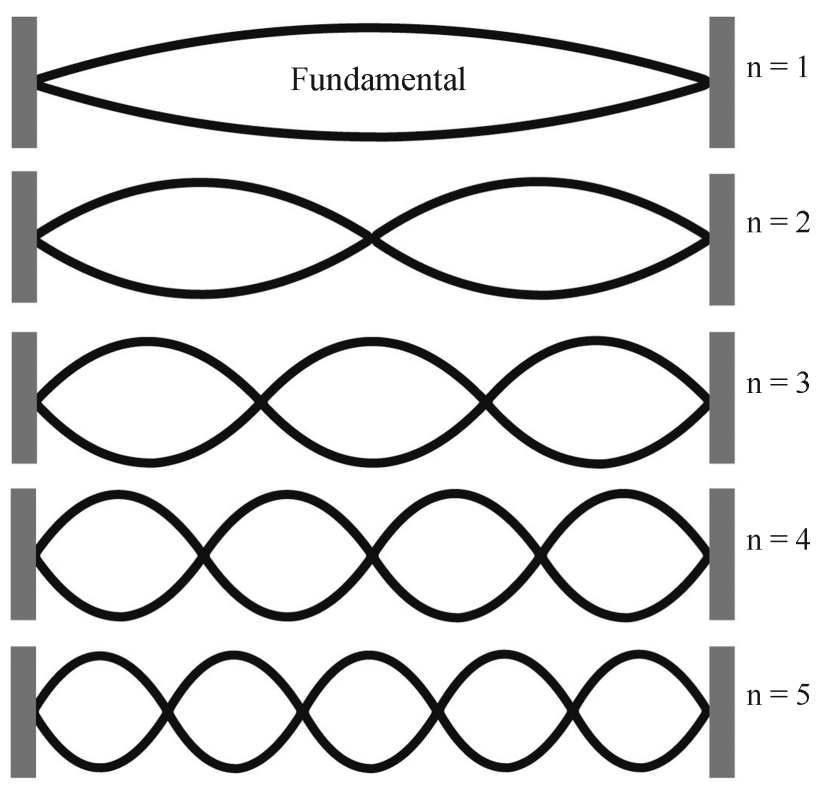

Figura 2 - Harmônicos em uma onda estacionária.

A frequência da onda estacionária em uma corda pode ser obtida pela equação

$$
f=\frac{v}{\lambda}=\frac{n v}{2 L},
$$

onde $v$ é a velocidade de propagação da onda através da corda. Outra equação importante no estudo das ondas estacionária é a que relaciona a velocidade $v$, a tração $T$ aplicada na corda e a densidade linear da corda $\mu$, resultando em

$$
v=\sqrt{\frac{T}{\mu}} .
$$

\section{Análise experimental}

Na proposta de experimento para estudo das ondas estacionárias em uma corda aqui apresentada utilizamos uma configuração já conhecida [3], mas substituímos o gerador de sinais por um celular smartphone (Fig. 1). O uso de celulares do tipo smartphone é bem comum entre nossos estudantes e daí surgiu o seguinte questionamento: $\mathrm{O}$ celular poderia ser utilizado como um gerador de sinais mantendo uma eficiência próxima a de um gerador comercial? Para responder a esse questionamento, inicialmente, pesquisamos aplicativos gratuitos para smatphones na loja online Google Play que funcionassem como geradores de sinais. O aplicativo denominado "PA Tone" [6] foi escolhido e instalado no

\footnotetext{
${ }^{2}$ As Refs. [3] e [5] trazem um aprofundamento teórico sobre ondas estacionárias em uma corda e a dedução das fórmulas aqui apresentadas
} 
smartphone Samsung Galaxy duos com sistema operacional Android 4.2.2 para testes. Foram realizados dois tipos de testes iniciais com o celular para verificar a viabilidade de uso do "PA Tone" como gerador de sinais, tais como:

- A análise do comportamento da forma da onda senoidal gerada na saída de áudio do celular, à medida que ocorria uma variação na frequência;

- Aferição da frequência do sinal gerado com o uso de um osciloscópio, para verificar se os valores na saída de áudio do dispositivo são idênticos ou muito próximos aos indicados pelo aplicativo.

Para a realização desses testes utilizamos um osciloscópio modelo DSO1112A da Agilent Technologies. O aplicativo "PA Tone" gera um sinal senoidal que é liberado pela saída de áudio do celular. Um conector P2 é utilizado para fazer a conexão entre a saída de áudio e os equipamentos de medida. Esse sinal é de baixa amplitude e antes de ser analisado sofre uma amplificação. Utilizamos o amplificador modelo KB10 da Comkitel (10 Watts RMS). Após o sinal ser amplificado analisamos o sinal através do osciloscópio. Um sinal de referência senoidal fornecido por um gerador comercial (Tektronix AFG, modelo 3021B) foi usado em outro canal do osciloscópio. O teste constituiu em variar a frequência através do celular e observar o padrão na forma de onda pelo osciloscópio. O osciloscópio foi programado para capturar a tela durante $10 \mathrm{~s}$, sendo que um frame era registrado a cada $1 \mathrm{~s}$. Portanto, após $10 \mathrm{~s}$ tivemos 10 registros da tela do osciloscópio, para cada uma das frequências analisadas. Realizamos esse procedimento com 10 valores de frequência, iniciando em $10 \mathrm{~Hz}$ e terminando em $100 \mathrm{~Hz}$, com intervalo de $10 \mathrm{~Hz}$ entre uma medida e outra.

Após os testes iniciais com o smartphone foi realizado um experimento para comparar o desempenho do aparelho com outras fontes geradoras de sinais senoidais como o software Audio SweepGen [7] e o gerador de sinais comercial Tektronix AFG modelo 3021B. O procedimento experimental constituiu em obter o valor da densidade linear da corda a partir de um gráfico de $T$ (tração na corda) contra $v^{2}$ (velocidade na corda ao quadrado). Nesse tipo de gráfico, o coeficiente angular da reta que melhor se ajusta aos pontos obtidos experimentalmente deve ser numericamente igual à densidade linear da corda.

Na montagem do experimento (Fig. 1) foram utilizados os seguintes componentes:

- Gerador de sinais;

- Amplificador de sinais;

- Fio (corda) de algodão de densidade linear medida $\mu=(4,73 \pm 0,12) x 10^{-4} \mathrm{~kg} / \mathrm{m}$;
- Pesos com massas conhecidas $\left(m_{1}=57 \mathrm{~g}, m_{2}=\right.$ $67,52 \mathrm{~g}, m_{3}=79,27 \mathrm{~g}, m_{4}=89,60 \mathrm{~g}$ e $m_{5}=$ $107 \mathrm{~g})$;

- Oscilador Mecânico (alto-falante com uma pequena haste acoplado em seu centro, similar aos usados em caixas de som para computadores ou de sistemas de som de automóveis);

- Software para geração de gráficos OriginLab versão 8.0 .

O procedimento experimental constituiu em colocar um disco de cobre de massa conhecida tracionando uma das extremidades do fio. A distância entre o centro da haste do alto-falante, onde se produziu a perturbação no fio, e o centro da roldana foi de $90 \mathrm{~cm}$. Com a corda tracionada por uma das massas, o oscilador mecânico (alto-falante), localizado na extremidade oposta, perturbou a corda executando movimento harmônico simples (MHS)

vertical de pequena amplitude. Essa perturbação tem a frequência controlada pelo gerador de funções produzindo sinal senoidal. Para observar os padrões de ondas estacionárias na corda foi necessário variar a frequência no gerador de sinais até que se observasse a formação dos harmônicos. O experimento foi realizado inicialmente utilizando o gerador comercial de funções Tektronix AFG (modelo 3021B) para obter os valores das frequências para cada padrão de onda estacionária observada na corda $(0,5 \lambda, 1 \lambda, 1,5 \lambda$ e $2 \lambda)$. Os valores das frequências dos harmônicos foram obtidos para diferentes massas tensionando a corda.

O mesmo procedimento experimental foi repetido utilizando os softwares SweepGen (Fig. 3a) e "PA Tone" (Fig. 3b) na geração de sinais senoidais. Como no procedimento anterior, variamos a frequência através do software até obter os mesmos harmônicos.

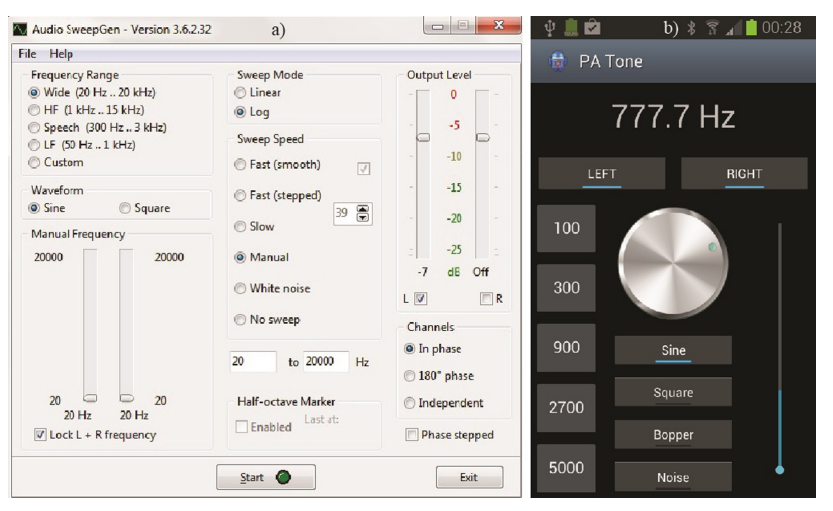

Figura 3 - (a) Visão do Software SweepGen para computadores. (b) Visão do aplicativo "PA Tone" para smartphones com sistema operacional androide.

\section{Resultados}

O sinal gerado pelo aplicativo "PA Tone" e captado através da saída de áudio do celular foi amplificado 
e analisado através de um osciloscópio. O comportamento da forma de onda foi observado para vários valores de frequência e o padrão senoidal manteve-se inalterado.

Os valores das frequências indicadas pelo aplicativo foram aferidos através do osciloscópio, e estão indicados na Tabela 1. Os valores de $\Delta f_{m a ́ x}$ referem-se à máxima diferença, no intervalo de $10 \mathrm{~s}$, entre os valores da frequência indicada pelo aplicativo "PA Tone" e registrada pelo osciloscópio $\left(f_{\text {oscil }}\right)$.

Os softwares testados apresentaram um bom desempenho quando comparados com o equipamento comercial. A Tabela 2 traz alguns dados obtidos com os 3 geradores de sinais utilizados e para diferentes valores de $n$. O aplicativo "PA Tone" apresentou um melhor desempenho na determinação da densidade linear do fio em relação ao software SweepGen. Isso pode ser explicado, em parte, pelo fato do SweepGen não permitir ajustar determinados valores de frequência em sua versão gratuita.

\section{Discussões e conclusões}

A reprodução deste experimento utilizando os softwares, possivelmente, não apresentará grandes dificuldades, mas alguns aspectos técnicos devem ser observados para que tudo funcione corretamente. O sinal captado na saída de áudio do notebook com o SweepGen tem maior amplitude do que a saída de áudio do celular com o "PA Tone", consequentemente uma pequena amplificação do sinal já é suficiente para que o SweepGen funcione adequadamente. Esta amplificação pode ser conseguida utilizando os amplificadores de caixinhas de som para computador, como já sugerido por outros autores. O problema da amplificação para ambos os softwares testados pode ser resolvido utilizando o am- plificador KB10 da Comkitel, que tem um baixo custo e pode ser encontrado facilmente em lojas de componentes eletrônicos ou pela internet.

O software Audio SweepGen tem algumas limitações em sua versão gratuita, por exemplo, determinadas frequências não são permitidas pelo software, acarretando em uma fonte de erro e, consequentemente, imprecisão nos valores obtidos para as velocidades das ondas na corda. O registro para a versão completa do SweepGen pode ser obtida pela internet e custa cerca de US\$ 47. O aplicativo "PA Tone" pode ser obtido de forma gratuita pela loja virtual Play Store da Google, para aplicativos de celular, e não tem o problema de "bloqueio" de determinados valores de frequência.

No experimento foi utilizado um alto-falante de $8 \Omega$ e $5 \mathrm{~W}$ com uma pequena haste de plástico colada com cola araldite em seu centro. Pela Tabela 2 podemos observar que os erros encontrados na determinação da densidade linear do fio são maiores para $n=2$, não importando o tipo de gerador de sinais utilizado. Após alguns testes, verificou-se que o componente responsável por essa fonte de erro foi o alto-falante. Com a substituição do alto-falante por outro de $8 \Omega$ e $40 \mathrm{~W}$, mantendo o mesmo amplificador já citado, foram obtidos erros menores no valor da densidade linear da corda para $n=2$, como: $0,84 \%$ para o "PA Tone", 0,21\% para Audio SweepGen e de 1,90\% para o Tektronix AFG. O software SweepGen apresenta uma boa precisão para valores de n de 1 a 3 , contudo, com valores acima de 3 , o "bloqueio" de frequências do software, em sua versão gratuita, dificulta a determinação das frequências onde observamos os harmônicos na corda. O alto-falante de $40 \mathrm{~W}$ exige uma maior amplificação e, possivelmente, a baixa amplificação de caixas de som para computador não seria suficiente para um funcionamento adequado do experimento.

Tabela 1 - Frequências dos sinais gerados pelo celular e aferidas com um osciloscópio.

\begin{tabular}{llllllllllll}
\hline $\begin{array}{l}\text { Frequência indicada pelo aplicativo "PA } \\
\left(f_{\text {patone }}\right) \text { em hertz }\end{array}$ & Tone" & 10 & 20 & 30 & 40 & 50 & 60 & 70 & 80 & 90 & 100 \\
\hline$\Delta f_{\max }=\left|f_{\text {oscil. }}-f_{\text {patone }}\right|$ & & 0 & 1 & 0,6 & 1 & 1 & 1 & 1 & 3 & 6 & 4 \\
\hline
\end{tabular}

Tabela 2 - Valores da densidade linear obtidos com diferentes geradores de sinais.

\begin{tabular}{lcll}
\hline Gerador de sinal & N. de harmônicos & $\begin{array}{l}\text { Densidade linear obtida grafica- } \\
\text { mente }(\mathrm{kg} / \mathrm{m})\end{array}$ & $\begin{array}{l}\text { Coeficiente } \\
\text { relação linear }\left(\mathrm{R}^{2}\right)\end{array}$ \\
\hline Tektronix AFG, & $\begin{array}{l}\text { Erro relativo da den- } \\
\text { sidade linear }(\%)\end{array}$ & 0,9999 \\
modelo 3021B & 1 & $(4,86 \pm 0,02) \times 10^{-4}$ & 0,9996 \\
& 2 & $(5,11 \pm 0,05) \times 10^{-4}$ & 0,9994 \\
& 3 & $(4,79 \pm 0,05) \times 10^{-4}$ & 0,9999 \\
Programa Audio & 4 & $(4,63 \pm 0,02) \times 10^{-4}$ & 0,9987 \\
Sweep Gen & 1 & $(5,08 \pm 0,06) \times 10^{-4}$ & 0,9993 \\
& 2 & $(4,87 \pm 0,09) \times 10^{-4}$ & 0,9979 \\
Aplicativo "PA Tone" & 3 & $(4,67 \pm 0,02) \times 10^{-4}$ & 0,9998 \\
& 4 & $(4,69 \pm 0,01) \times 10^{-4}$ & 0,9999 \\
& 1 & $(5,02 \pm 0,04) \times 10^{-4}$ & 0,9996 \\
\end{tabular}


A configuração do experimento para estudo da onda estacionária em uma corda utilizando o aplicativo "PA Tone" pode, portanto, substituir o gerador de sinais comercial, permitindo que o experimento seja reproduzido em laboratórios a um baixo custo e com precisão similar. A montagem do experimento pode ser considerada simples e os materiais utilizados podem ser encontrados com facilidade. Os celulares modernos, do tipo smartphone, apresentam uma série de sensores e aplicativos que podem ser explorados, podendo ser considerados um recurso didático a mais disponível ao professor de física.

\section{Referências}

[1] S.E. Duarte, Cad. Bras. Ens. Fís. 29, 525 (2012).

[2] M.A. Cavalcante e C.R.C Tavolaro, In: Anais do IX Encontro Nacional de Pesquisa em Ensino de Física, Jaboticatubas, MG, 2004. Disponível em http://www.sbf1.sbfisica.org.br/eventos/ epef/ix/atas/posteres/po51-39.pdf. Acesso em $22 / 6 / 2014$.

[3] G.I. Mello, Física na Escola 8(2), 31 (2007).

[4] M.A. Cavalcante, R. Peçanha e A.C. Teixeira, Revista Brasileira de Ensino de Física 35, 3502 (2013).

[5] R. Guerra, Ondas Estacionárias Disponível em http://w3.ualg.pt/ rguerra/Acustica/ estacionarias.pdf. Acesso em 17/6/2014.

[6] PA Tone. Descrição do aplicativo. Disponível em https://play.google.com/store/apps/details? id=com.dutchmatic. patone\&hI=pt_BR. Acesso em $20 / 6 / 2014$.

[7] Audio SweepGen. Disponível em http://www. satsignal.eu/software/audio.html. Acesso em $8 / 3 / 2014$. 